\title{
ON THE COMPUTATION OF PERIODIC ORBITS AND INVARIANT SURFACES OF $4 D$-SYMPLECTIC MAPPINGS
}

\author{
M.N. VRAHATIS, T.C. BOUNTIS \\ University of Patras, Department of Mathematics, GR-26110 Patras, Greece \\ AND M. KOLLMANN \\ Institute for Theoretical Physics, University of Amsterdam \\ Valckenierstraat 65, 1018 Amsterdam, the Netherlands
}

\begin{abstract}
An efficient numerical method is applied to a 4-D symplectic map of physical interest. Sequences of periodic orbits of very high period are obtained, whose rotation numbers approach two rationally independent ratios. Thus we are able to investigate the connection between the arrangement and stability of these orbits and the "morphology" of nearby invariant surfaces "surrounding" large regions of regular motion in 4-D space.
\end{abstract}

\section{Introduction}

As is well known, $N-\mathrm{D}$ mappings of the form :

$$
X^{\prime}=T(X), \quad X=\left(x_{1}, x_{2}, \ldots, x_{N}\right)
$$

can model conservative dynamical systems, if the determinant of the Jacobian of the map is unity, i.e. $\left|\operatorname{det} J_{T}\right|=1$, or dissipative ones if $\left|\operatorname{det} J_{T}\right|<1,[1,3]$. We say that $X=\left(x_{1}, x_{2}, \ldots, x_{N}\right)$ is a fixed point of $T$ of order $p$ (or a periodic orbit of period $p$ ), if :

$$
X=T^{p}(X) \equiv \underbrace{T(T(\cdots(T(X)) \cdots))}_{p \text { times }}, \quad p=1,2,3, \ldots
$$

In general, it is difficult to find in the literature efficient methods for computing periodic orbits of high period for $N(>2)$-Dimensional maps. Traditional iterative schemes, such as Newton's method and related algorithms [10], often fail since they converge to the same fixed point almost independently of the initial guess, while there may exist several other fixed points nearby, which are all desirable for the applications. Also, these methods are generally affected by the map evaluations taking large values, or may break down due to the nonexistence of derivatives or poorly behaved partial derivatives near fixed points [10].

We apply an efficient method for rapidly computing periodic orbits of $N-\mathrm{D}$ maps to any desired accuracy. This method exploits topological degree theory to provide a criterion for the existence of a periodic orbit of an iterate of the map within a given region.

Our method, referred to here as the $\mathrm{CP}$ (characteristic polyhedron)-criterion, is especially useful for the computation of high period orbits and is quite efficient, since the only computable information required is the algebraic sign of the components of the mapping. Thus, it is not significantly affected by the unavoidable inaccuracies of the calculations in the neighborhood of unstable periodic orbits. 
Here, this method is applied to a 4-D symplectic map representing an interesting model, which is of direct relevance to the dynamics of particle beams passing repeatedly through FODO cells of magnetic focusing elements [5, 4].

\section{The $\mathbf{C P}$-criterion}

We give a criterion for the existence of a periodic orbit within a given region of the phase space of the system. This criterion is based on the construction of a sequence of "characteristic polyhedra" within a scaled translation of the unit cube, $[12,15,13]$.

The problem of finding periodic orbits of nonlinear mappings $T: \mathcal{D} \subset \mathbb{R}^{n} \rightarrow \mathbb{R}^{n}$ of period $p$ amounts to finding fixed points $X^{\star}=\left(x_{1}^{\star}, x_{2}^{\star}, \ldots, x_{n}^{\star}\right) \in \mathcal{D}$ of $T^{p}$ which satisfy the following system of equations :

$$
F_{n}(X)=\mathcal{O}_{n}=(0,0, \ldots, 0),
$$

with $F_{n}=\left(f_{1}, f_{2}, \ldots, f_{n}\right)=T^{p}-I_{n}$, where $I_{n}$ is the $n \times n$ identity matrix.

Let us define a characteristic polyhedron by constructing the $2^{n} \times n$ matrices $\mathcal{M}_{n}$ whose rows are formed by all possible combinations of $-1,1$. The $n$-polyhedron $\Pi^{n}=$ $\left\langle\Upsilon_{1}, \Upsilon_{2}, \ldots, \Upsilon_{2^{n}}\right\rangle$ in $\mathbb{R}^{n}$ is called a characteristic polyhedron $(C P)$ relative to $F_{n}$ if the matrix of signs associated with $F_{n}$ and $\Pi^{n}, \mathcal{S}\left(F_{n} ; \Pi^{n}\right)$, is identical with the $n$-complete matrix $\mathcal{M}_{n}$. In other words, the $2^{n}$ vertices of $\Pi^{n}$ obtain every combination of \pm 1 .

If $\Pi^{n}$ is a CP then, under suitable assumptions on the boundary of $\Pi^{n}$ the value of the topological degree of $F_{n}$ at $\mathcal{O}_{n}$ relative to $\Pi^{n}$ is nonzero which implies the existence of a periodic orbit inside $\Pi^{n}$. For a detailed description of how to construct a CP and locate a desired periodic orbit see $[12,15,13]$.

The generalized bisection method, used in combination with the $\mathrm{CP}-$ criterion outlined above, bisects a CP, in such a way that the new refined $n$-polyhedron is also a CP. To do this, one computes the midpoint of a proper 1-simplex (edge) of $\Pi^{n}$ and uses it to replace that vertex of $\Pi^{n}$ for which the vectors of their signs are identical (see $[12,15,14,13]$ for details). Finally, the number $B$ of characteristic bisections of the edges of a $\Pi^{n}$ required to obtain a new refined CP, $\Pi_{\star}^{n}$, whose longest edge length, $\Delta\left(\Pi_{\star}^{n}\right)$, satisfies $\Delta\left(\Pi_{\star}^{n}\right) \leq \varepsilon$, for some $\varepsilon \in(0,1)$, is given by $B=\left\lceil\log _{2}\left(\Delta\left(\Pi^{n}\right) \varepsilon^{-1}\right)\right\rceil,[12]$.

\section{Periodic Orbits and Invariant Surfaces}

Let us now proceed to illustrate the method on the following 4-D mapping :

$$
T:\left(\begin{array}{l}
x_{1}^{\prime} \\
x_{2}^{\prime} \\
x_{3}^{\prime} \\
x_{4}^{\prime}
\end{array}\right)=\left(\begin{array}{cccc}
\cos \omega_{1} & -\sin \omega_{1} & 0 & 0 \\
\sin \omega_{1} & \cos \omega_{1} & 0 & 0 \\
0 & 0 & \cos \omega_{2} & -\sin \omega_{2} \\
0 & 0 & \sin \omega_{2} & \cos \omega_{2}
\end{array}\right)\left(\begin{array}{c}
x_{1} \\
x_{2}+x_{1}^{2}-x_{3}^{2} \\
x_{3} \\
x_{4}-2 x_{1} x_{3}
\end{array}\right)
$$

for two generally different values of the "linear" frequencies $\omega_{1}, \omega_{2}$. In order to compute a periodic orbit of (2) with period $p$, we have to solve the corresponding System (1), for any given $p$ and $n=4$. The stability of these orbits is determined by the eigenvalues of the return Jacobian matrix $J_{T}^{(p)},[7,3]$. As is known, for the $p$-periodic orbit to be stable the eigenvalues of $J_{T}^{(p)}$ must all lie on the unit circle. In that case we call the orbit elliptic-elliptic (EE). If the first two eigenvalues $\lambda_{1}, \lambda_{2}$ (in the conventional ordering of orthogonal eigenvectors) are on the unit circle, while $\lambda_{3}, \lambda_{4}$ are real (with $\left|\lambda_{3}\right|>1$, say 
and $\left|\lambda_{4}\right|<1$ ) we call the orbit elliptic-hyperbolic (EH) or hyperbolic-elliptic (HE) if $\lambda_{1}, \lambda_{2}$ are interchanged with $\lambda_{3}, \lambda_{4}$. If all $\lambda_{i}$ are real (with $\left|\lambda_{1}\right|>1,\left|\lambda_{3}\right|>1$, say, and $\left|\lambda_{2}\right|<1$, $\left.\left|\lambda_{4}\right|<1\right)$ the orbit is termed hyperbolic-hyperbolic $(\mathrm{HH})$, while, if all $\lambda_{i}$ are complex, with $\left|\lambda_{1}\right|=\left|\lambda_{2}\right|>1$, say, and $\left|\lambda_{3}\right|=\left|\lambda_{4}\right|<1$ we call it complex unstable (CU).

Using our method, we have been able to make some interesting observations about the periodic orbits of (2): The first one concerns the arrangement of different orbits of the same period, in 4-D space, according to their stability. The second one is connected with the existence of smooth invariant surfaces which extend around the origin, "enveloping" these periodic orbits in a way reminiscent of the case of area-preserving $2-\mathrm{D}$ maps $[7,8,6,9]$.

Let us fix the frequencies of our map $q_{x}=\omega_{1} / 2 \pi=0.168$ and $q_{y}=\omega_{2} / 2 \pi=0.201$ close to the resonance : $\left(\sigma_{1}, \sigma_{2}\right)=(1 / 6,1 / 5)$ where $\sigma_{1}, \sigma_{2}$ are the winding numbers of the orbit around the origin of the $\left(x_{1}, x_{2}\right)$ and $\left(x_{3}, x_{4}\right)$ planes respectively. As the lowest common denominator of these fractions is 30 , we expect to find that (2) possesses, at these parameter values, periodic orbits of period 30 . Indeed, such orbits were easily located and computed, using the methods of Section 2. The distinction between EH and HE (and the corresponding ordering of the eigenvalues) follows from ordering the eigenvectors according to the magnitude of their projections in the $x_{1}-x_{2}$ and $x_{3}-x_{4}$ subspaces, see Fig. 1a.

If we now perturb the initial conditions of one of the $\mathrm{EH}$ points, the orbit begins to wander away "enveloping" also the EE in each group, forming six 2-D thin tori, whose projection in 3-D space is shown in Fig. 1b. As the perturbation increases, the size of these 2-D toroidal "islands" grows (Fig. 1c), until they join into one 2-D surface that "envelops" them all and extends around the origin, as shown in Fig. 1d. This appears to be a rotational invariant surface, analogous to the ones studied by Greene and MacKay for 2-D area-preserving maps [7,8]. Unlike the 2-D case, however, such surfaces do not divide the 4-dimensional phase space in disjoint regions. This is why Arnol'd diffusion phenomena in these maps are always possible $[5,4]$.

Thus, we decided to take a closer look at these surfaces approximating them with periodic orbits in the following way : Following a method introduced by Greene [7] for $2-\mathrm{D}$ maps, we have located periodic orbits, whose (rational) rotation numbers $\sigma_{n}^{(i)}$ limit on two independent irrational rotation numbers $\sigma_{1}, \sigma_{2}$ of an invariant surface, as : $\sigma_{i}=$ $\lim _{n \rightarrow \infty} \sigma_{n}^{(i)}=\lim _{n \rightarrow \infty}\left(p_{n}^{(i)} / q_{n}^{(i)}\right)$. The period of these orbits will be sought as the product, or the lowest common multiple of the denominators of their winding numbers $\sigma_{n}^{(i)}$. Let us choose, for example $\sigma_{1}=(\sqrt{5}-1) / 2=0.618033989 \ldots, \sigma_{2}=\sqrt{2}-1=0.414213562 \ldots$, as the winding numbers of the invariant surface we wish to approximate.

We shall look for periodic orbits whose rotation numbers given in Table 1 are rational approximants of $\sigma_{1}, \sigma_{2}$ from their continued fraction expansions. Following such sequences

TABLE 1. Rational approximants in the continued fraction expansions of the quadratic irrationals $\sigma_{1}$ and $\sigma_{2}$ and the full period of the corresponding periodic orbit.

\begin{tabular}{ccccccccc}
\hline$\sigma_{n}^{(1)}=\frac{p_{n}^{(1)}}{q_{n}^{(1)}}$ & $\frac{1}{1}$ & $\frac{1}{2}$ & $\frac{2}{3}$ & $\frac{3}{5}$ & $\frac{5}{8}$ & $\frac{8}{13}$ & $\frac{13}{21}$ & $\frac{21}{34}$ \\
\hline$\sigma_{n}^{(2)}=\frac{p_{n}^{(2)}}{q_{n}^{(2)}}$ & $\frac{1}{2}$ & $\frac{2}{5}$ & $\frac{5}{12}$ & $\frac{12}{29}$ & $\frac{29}{70}$ & $\frac{70}{169}$ & $\frac{169}{408}$ & $\frac{408}{985}$ \\
\hline Full Period & 2 & 10 & $36(12)$ & 145 & $560(280)$ & $2197(169)$ & $8568(2856)$ & 33490 \\
\hline
\end{tabular}



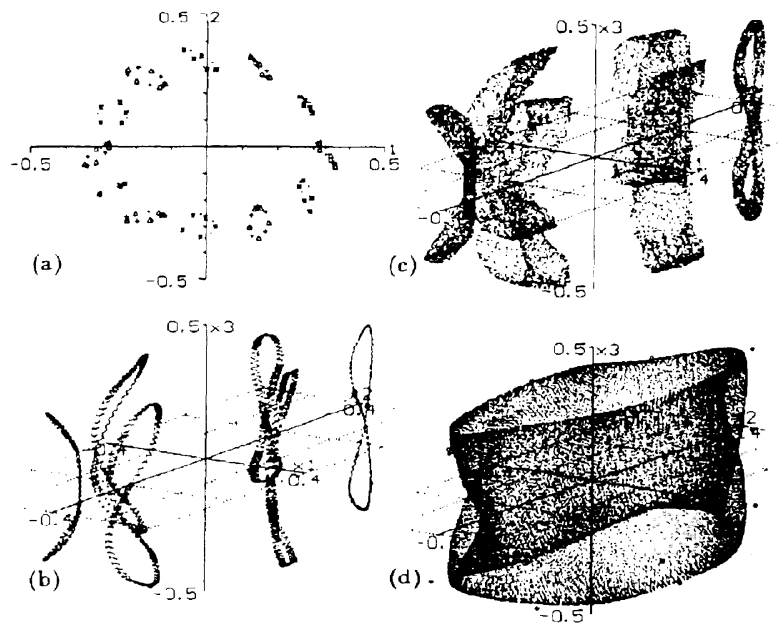

Figure 1. (a) Constellation of 12 groups consisting of 10 points of period-30 orbits for $q_{x}=0.168$, $q_{y}=0.201$. Six of these groups are composed, alternatingly of five $\operatorname{EE~}(\triangle)$ and five $\mathrm{EH}(+)$ points and are separated by six other 10 -point groups of alternating five $\mathrm{HE}(\bullet)$ and five $\mathrm{HH}(*)$ points. (b)-(d) A surrounding invariant torus appears, by increasing the perturbation of one of the EH points.
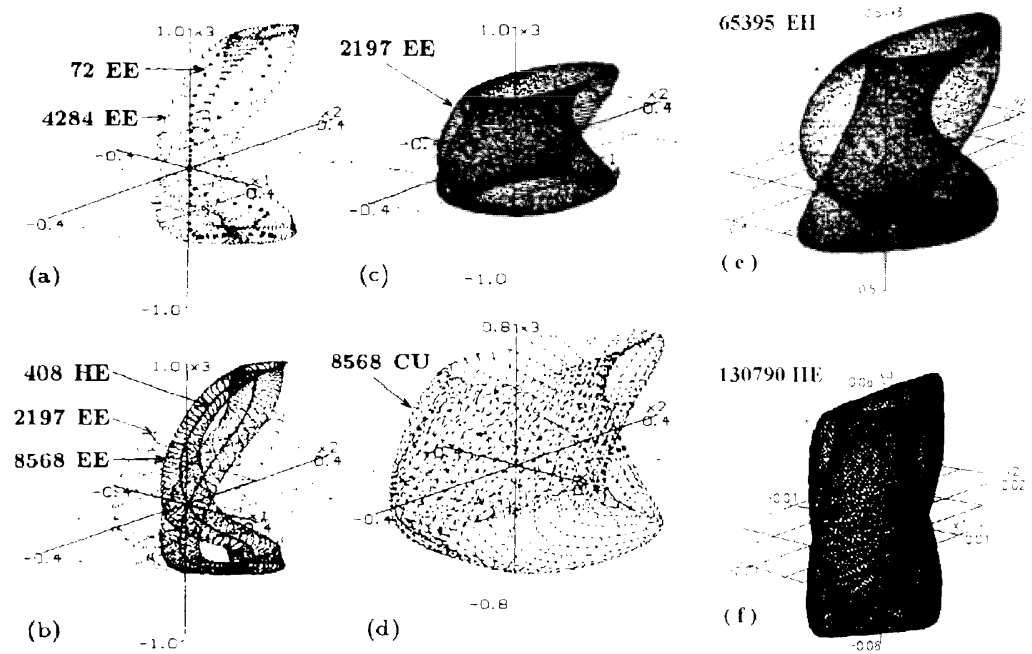

Figure 2. Periods and stability of various periodic orbits for the frequencies (a)-(d) $q_{x}=0.61903$, $q_{y}=0.4152$ (e), (f) $q_{x}=2 / 3, q_{y}=5 / 12$. In (c) the 2197 period orbit is perturbed and (d) is after $5 \cdot 10^{5}$ iterations.

of rotation numbers, with $q_{x}=\omega_{1} / 2 \pi=\sigma_{1}+10^{-3}$ and $q_{y}=\omega_{2} / 2 \pi=\sigma_{2}+10^{-3}$, we have succeeded in computing the corresponding periodic orbits for periods up to a hundred thousand, within an accuracy of $\varepsilon=10^{-15}$. In Fig 2 we display some of these orbits, together with their stability properties. We have computed, of course, for each period, all 
the orbits with different stability characteristics. The eigenvalues $\lambda_{i}$ of all orbits indicate that these orbits have either just turned unstable, (if some $\left|\lambda_{i}\right|>1$ in the EH, HE and CU cases), or are very close to doing so, in the EE cases, where all $\lambda_{i}$ 's are still on the unit circle. In fact, for the EE case, by increasing the period, the $\left|\lambda_{i}\right|$ tend closer to one. (An analytical approach to the structure of resonances in phase space, for a class of nearintegrable mappings, has been very recently presented in [11]).

All this serves to strengthen the argument that an analogue of Greene's 2-D criterion may hold for 4-D symplectic maps as well. However, the situation in 4-D is somewhat less clear, since the invariant surface we are approximating at every stage is not the same for all periodic orbits. Thus, even though there are some evident similarities with the 2D case, we cannot claim that a simple analogue of Greene's criterion holds for the type of 4-D symplectic mapping considered in this paper. On the other hand, in mapping examples which do satisfy the twist condition, there is evidence that Greene's criterion can be generalized to higher dimensions [2].

\section{References}

1. Birkhoff, G.D. (1917) Dynamical systems with two degrees of freedom, Trans. Amer. Math. Soc., 18, pp. 199-300.

2. Bollt, E.M. and Meiss, J.D. (1992) Breakup of invariant tori for the four dimensional semi-standard map, in Program in applied mathematics, PAM \#134, University of Colorado, Boulder.

3. Bountis, T.C. and Helleman, R.H.G. (1981) On the stability of periodic orbits of two-dimensional mappings, J. Math. Phys., 22, pp. 1867-1877.

4. Bountis, T.C. and Kollmann, M. (1994) Diffusion rates in a 4-dimensional mapping model of accelerator dynamics, Physica D, 71, pp. 122-131.

5. Bountis, T.C. and Tompaidis, S. (1991) Strong and weak instabilities in a 4D mapping model of FODO cell dynamics, in Future Problems in Nonlinear Particle Accelerators, eds. Turchetti, G. and Scandale, W. (World Scientific), pp. 112-127.

6. Falcolini, C. and de la Llave, R. (1992) A rigorous partial justification of Greene's criterion, J. Stat. Phys., 67, pp. 609 643.

7. Greene, J.M. (1979) A method for determining a stochastic transition, J. Math. Phys., 20, pp. 1183 1201.

8. MacKay, R.S. (1982) Renormalization in area preserving maps, Ph.D. Dissertation, Princeton University and (1994) 2nd World Scientific; see also several papers in (1986) Hamiltonian Dynamical systems, eds. Mackay, R.S. and Meiss, J.D., Adams Hilger, Bristol.

9. MacKay, R.S. (1992) On Greene's residue criterion, Nonlinearity, 5, pp. 161-187.

10. Ortega, J.M. and Rheinboldt, W.C. (1970) Iterative solution of nonlinear equations in several variables (Academic Press, New York).

11. Todesco, E. (1994) Analysis of resonant structures of four-dimensional symplectic mappings, using normal forms, Phys. Rev. E, 50, pp. 4298-4301.

12. Vrahatis, M.N. (1988) Solving systems of nonlinear equations using the nonzero value of the topological degree, ACM Trans. Math. Software, 14, pp. 312-329.

13. Vrahatis, M.N. (1995) An efficient method for locating and computing periodic orbits of nonlinear mappings, J. Comp. Phys., 119, pp. 105-119.

14. Vrahatis, M.N. and Bountis, T.C. (1994) An efficient method for computing periodic orbits of conservative dynamical systems, in International conference on Hamiltonian mechanics, Integrability and chaotic behavior, ed. Seimenis, J. (Plenum Press, New York), pp. 261-274.

15. Vrahatis, M.N., Servizi, G., Turchetti, G. and Bountis, T.C. (1993) A procedure to compute the periodic orbits and visualize the orbits of a 2D map, CERN SL/93-06 (AP). 